\title{
FLVCR1 promotes the proliferation and tumorigenicity of synovial sarcoma through inhibiting apoptosis and autophagy
}

\author{
CHANGLIANG PENG ${ }^{1}$, YAN SONG ${ }^{2}$, WEI CHEN ${ }^{3}$, XIAOYING WANG $^{4}$, XIAOLI LIU $^{5}$, FANG WANG $^{6}$, \\ DONGJIN WU ${ }^{1}$, SHENGZHONG MA ${ }^{1}$, XIUWEN WANG ${ }^{1}$ and CHUNZHENG GAO ${ }^{1}$
}

\author{
Departments of ${ }^{1}$ Orthopaedics and ${ }^{2}$ Nephrology, The Second Hospital of Shandong University, Jinan, Shandong 250033; \\ ${ }^{3}$ Beijing Institute of Pharmacology and Toxicology, Beijing 100850; Departments of ${ }^{4}$ Pathology and ${ }^{5}$ Hematology; \\ ${ }^{6}$ Clinical Laboratory, The Second Hospital of Shandong University, Jinan, Shandong 250033, P.R. China
}

Received December 20, 2017; Accepted February 12, 2018

DOI: $10.3892 /$ ijo.2018.4312

\begin{abstract}
Feline leukemia virus subgroup $\mathrm{C}$ receptor 1 (FLVCR1) has been reported to have a crucial role in variety of biological processes, including cell proliferation, cell death, apoptosis, oxidative stress response, cellular differentiation and metabolism. However, little is known about its role in synovial sarcoma (SS). In the current study, FLVCR1 expression was analyzed in two SS cell lines (SW982 and HS-SY-II), and in eight SS tissues and paired adjacent non-tumor tissues using reverse transcription-quantitative polymerase chain reaction, western blot analysis and immunohistochemistry. Lentivirus-mediated short hairpin RNAs were used to knock down FLVCR1 expression in SW982 and HS-SY-II cells. The effects of FLVCR1 knockdown on the cell proliferation, clonogenicity, cell cycle and apoptosis in SS cells were evaluated by MTT, colony formation assay, flow cytometry analysis, western blotting and in vivo tumorigenesis in nude mice. In the current study, gene expression of FLVCR1 was upregulated in SS cell lines (SW982 and HS-SY-II) and SS tissues from patients. The protein levels of FLVCR1 in SS tissues were also significantly higher than in adjacent non-tumor tissues. Furthermore, suppressing the expression of FLVCR1 in SS cells using short hairpin RNA effectively attenuated cell proliferation, colony formation and impaired the cell cycle, and also significantly induced apoptosis and autophagy. In accordance with this, an in vivo tumorigenicity assay in mice demonstrated that
\end{abstract}

Correspondence to: Dr Changliang Peng, Department of Orthopedics, The Second Hospital of Shandong University, 247 Beiyuan Street, Jinan, Shandong 250033, P.R. China

E-mail: pengchangliangvip@163.com

Dr Yan Song, Department of Nephrology, The Second Hospital of Shandong University, 247 Beiyuan Street, Jinan, Shandong 250033, P.R. China

E-mail: sy23.y-ok@163.com

Key words: feline leukemia virus subgroup $\mathrm{C}$ receptor 1, synovial sarcoma, proliferation, tumorigenicity, autophagy suppression of FLVCR1 expression inhibited the growth of SS tumors implanted subcutaneously. Collectively, these results demonstrated that FLVCR1 may act as an oncoprotein, and have key roles in promoting proliferation and tumorigenicity of SS, and this may shed new light on finding novel therapeutic strategies against $\mathrm{SS}$.

\section{Introduction}

Synovial sarcoma (SS) is an aggressive malignant tumor of mesenchymal origin, with high risk of local recurrence and distant metastasis, and is also characterized by poor prognosis, high mortality and a poor curative rate $(1,2)$. SS frequently originates at the extremities of young adults and constitutes 10-20\% of all soft tissue sarcomas (3). Current therapy for SS comprises surgery with or without adjuvant chemotherapy and/or radiotherapy, which provides a good opportunity for healing of localized disease. However, early and late recurrences often occur $(4,5)$, and the 5-year survival rate is estimated to be only $27-55 \%$ (6). Mounting evidence indicates that certain proteins are dysregulated in primary tumors and are associated with the development and progression of SS (7-9). Understanding the specific functions and disclosing the underlying mechanisms of these proteins involved in the occurrence and development of SS may facilitate identification of effective targeting strategies for SS.

Feline leukemia virus subgroup C cellular receptor 1 (FLVCR1), as a member of the major facilitator superfamily transporters, has important roles in controlling the size of the cytoplasmic free-heme pool (10). There are two different isoforms of FLVCR1: FLVCR1a and FLVCR1b, the former is a plasma membrane heme exporter mediating heme export out of the cell $(11,12)$, and the latter resides in the mitochondria and is involved in heme trafficking between the mitochondria and cytosol (13). FLVCR1a is required for preventing hemorrhages and edema, whereas FLVCR1b regulates erythropoiesis by controlling mitochondrial heme efflux (10). Mercurio et al (14) reported that FLVCR1a is crucial for the proliferation of committed erythroid progenitors, and FLVCR1b is crucial for terminal differentiation. Additionally, Fiorito et al (13) demonstrated that the expression of FLVCR1 was regulated by hypoxia in human colorectal adenocarcinoma Caco 2 cells 
through hypoxia inducible factors $2 \alpha$ (HIF $2 \alpha)$ and v-ets avian erythroblastosis virus E26 oncogene homolog 1 (ETS1), thus demonstrating the role of FLVCR1 in hypoxia-stimulated processes, including erythropoiesis, vasculogenesis, and potentially heme absorption. FLVCR1 is reported to be involved in sensory neuron maintenance and posterior column ataxia, and retinitis pigmentosa $(15,16)$. Chiabrando et al (15) reported that FLVCR1 is important for the survival of neuroblastoma cells, and they further demonstrated that FLVCR1 is crucial in mediating oxidative stress and regulating the sensitivity to programmed cell death in several cell types, including erythroid progenitors, hepatocytes and intestinal cells (17-19). Together, these findings suggest that FLVCR1 may have vital roles in a variety of biological processes, including cell proliferation, cell death, apoptosis, oxidative stress response, cellular differentiation and metabolism.

In the current study, the expression of FLVCR1 was markedly elevated in SS cells and clinical SS samples. However, the biological roles of the overexpressed FLVCR1 in SS remain unknown. According to aforementioned roles of FLVCR1, we hypothesized that FLVCR1 may contribute to the occurrence and development of SS. By silencing FLVCR1 using specific short hairpin RNA (RNA), proliferation and tumorigenicity of SS cells were inhibited in vitro and in vivo. FLVCR1 promoted the proliferation and tumorigenicity of SS by regulating cytotoxic autophagy. To the best of our knowledge, these findings were the first to demonstrate that FLVCR1 functions as an oncoprotein during SS progression, which indicates that FLVCR1 may be a novel therapeutic target for SS.

\section{Materials and methods}

Tissue samples. Samples of human SS tissues and paired adjacent non-tumor tissues that were $5 \mathrm{~cm}$ away from the tumors were obtained from 8 patients histopathologically diagnosed with SS that underwent surgical resections between May 2014 and September 2017 at the Second Hospital of Shandong University (Jinan, China). None of the patients had received anticancer therapy prior to the sampling. Of 8 patients, 5 were male and 3 female. The age of the patients at the time of presentation ranged between 18 and 76 years (mean, 38.5 years). Fibroblast-like synovialcytes were isolated from 2 patients (1 male aged 65 years, 1 female aged 57 years) diagnosed with osteoarthritis who underwent surgical resections in July 2017. The samples were obtained with patients' informed consent. All research involving human participants have been approved by the Second Hospital of Shandong University ethics committees and in accordance with the Declaration of Helsinki.

Cell lines. The human HS-SY-II SS cell line was provided by Dr Yi Guo (University of California, Irvine, CA, USA), and cultured in Dulbecco's modified Eagle's medium (Gibco; Thermo Fisher Scientific, Inc., Waltham, MA, USA). Human SW982 (no. HTB-93) SS cell line was obtained from the American Type Culture Collection (Manassas, VA, USA), and propagated in L-15 medium (Gibco; Thermo Fisher Scientific, Inc.). Human fibroblast-like synovialcytes derived from patients with osteoarthritis were cultured in RPMI-1640 medium (Gibco; Thermo Fisher Scientific, Inc.). All the media were supplemented with $10 \%$ fetal bovine serum (Gibco;
Thermo Fisher Scientific, Inc.), $100 \mathrm{U} / \mathrm{ml}$ penicillin and $100 \mu \mathrm{g} / \mathrm{ml}$ streptomycin. Cells were cultured at $37^{\circ} \mathrm{C}$ under a $5 \% \mathrm{CO}_{2}$ atmosphere.

Immunohistochemistry. The immunohistochemical procedures were performed as previously reported $(20,21)$. Antibody against FLVCR1 (cat. no. PA5-61270) was purchased from Invitrogen (Thermo Fisher Scientific, Inc.). Briefly, $10 \%$ formalin-fixed, paraffin-embedded human SS or paired adjacent non-tumor samples were cut into $5-\mu \mathrm{m}$ sections, placed on glass slides, heated at $70^{\circ} \mathrm{C}$ for $30 \mathrm{~min}$, and then deparaffinized with xylene and ethanol. For antigen retrieval, the deparaffinized and rehydrated slides were heated in $10 \mathrm{mM}$ citrate buffer for $20 \mathrm{~min}$ in a microwave oven and allowed to cool for $20 \mathrm{~min}$ at room temperature. Slides were incubated with $3 \% \mathrm{H}_{2} \mathrm{O}_{2}$ in methanol for $15 \mathrm{~min}$ at room temperature to eliminate endogenous peroxidase activity. Then slides were incubated at $4^{\circ} \mathrm{C}$ overnight with antibody against the rabbit polyclonal anti-FLVCR1 antibody (1:50). Sections incubated with nonimmune rabbit serum $(1: 100$; cat. no. FK-MB594J; Shanghai Fanke Biotech Co., Ltd., Shanghai, China) in PBS instead of primary antibody served as negative controls. After incubation in a 1:2,000 dilution of secondary antibody (cat. no. ab6720; Abcam, Cambridge, MA, USA) for $4 \mathrm{~h}$ at room temperature, the slides were incubated with a streptavidin-peroxidase complex at room temperature for $20 \mathrm{~min}$. Immunohistochemistry staining was performed using 3,3'-diaminobenzidine Enhanced Liquid Substrate System tetrahydrochloride (cat. no. D3939; Sigma-Aldrich, Merck KGaA, Darmstadt, Germany) and counterstained with haematoxylin. Cells exhibiting positive staining on cell membranes and in the cytoplasm and nucleus were counted in at least 10 representative fields (in total 80 sections were used; magnification, $\mathrm{x} 200$ ) and the mean percentage of positive cells was calculated. Immunostaining was evaluated by two independent pathologists blinded to clinical characteristics and outcomes.

Reverse transcription-quantitative polymerase chain reaction (RT-qPCR). Total RNA was extracted using TRIzol reagent (Invitrogen; Thermo Fisher Scientific, Inc.), and RT was performed to cDNA using the M-MLV reverse transcriptase kit (Promega Corporation, Madison, WI, USA) in accordance to the manufacturer's instructions. The PCR primers for FLVCR1 and GAPDH were as follows: FLVCR1 forward, 5'-CCCACA GACCAAGAACC-3' and reverse, 5'-CCACCACATACAAA CCC-3'; GAPDH forward, 5'-TGACTTCAACAGCGACAC CCA-3 and reverse, 5'-CACCCTGTTGCTGTAGCCAAA-3'. qPCR was performed with the SYBR-Green Real-Time PCR assay kit (Takara Bio, Inc., Otsu, Japan) on an ABI PRISM 7300 Sequence Detection System (Applied Biosystems; Thermo Fisher Scientific, Inc.). The thermal cycling conditions were as follows: initial denaturation at $95^{\circ} \mathrm{C}$ for $10 \mathrm{~min}$, followed by 35 amplification cycles at $95^{\circ} \mathrm{C}$ for $15 \mathrm{sec}, 60^{\circ} \mathrm{C}$ for $1 \mathrm{~min}$. The level of GAPDH was used as an internal control. Fold changes in expression were calculated using the $2^{-\Delta \Delta \mathrm{Cq}}$ method (22).

Vectors and lentivirus infection. For depletion of FLVCR1, a human FLVCR1-targeting shRNA sequence was cloned into pGCSIL-green fluorescent protein (GFP) vector to generate 
pGCSIL-GFP-FLVCR1-RNAi, with target sequence 5'-CCC TGAAGAGTACTCCTATAA-3' (synthesized by Shanghai GeneChem Co., Ltd., Shanghai, China), or control scrambled shRNA (Lv-shCon sequence, 5'-TTCTCCGAACGTGTCA CGT-3'). Lentiviruses production and infection were performed as previously reported (7). Stable cell lines (SW982 and HS-SY-II) expressing FLVCR1 shRNAs were selected for 10 days with $0.5 \mu \mathrm{g} / \mathrm{ml}$ puromycin. At 3 weeks later, the cells were essentially $100 \%$ GFP positive, and processed for RT-qPCR and western blot.

Western blotting. Standard western blot assays were performed as described previously $(7,23)$. Briefly, cells were harvested in the NE-PER ${ }^{\text {TM }}$ Nuclear and Cytoplasmic Extraction Reagents (cat. no. 78833; Thermo Fisher Scientific, Inc.), and protein was quantified with Pierce ${ }^{\mathrm{TM}}$ BCA Protein Assay kit (cat. no. 23227; Pierce; Thermo Fisher Scientific, Inc.). Then, equal amounts of protein $(30 \mu \mathrm{g})$ were electrophoresed by $10 \%$ SDS-PAGE and transferred onto polyvinylidene fluoride membranes. Then membranes were blocked overnight at $4^{\circ} \mathrm{C}$ in $5 \%$ skim milk and incubated for $2 \mathrm{~h}$ at room temperature with the primary antibodies. The membranes were then washed with 1X TBS-Tween-20 three times and incubated with horseradish peroxidase-conjugated antibodies (1:10,000; cat. no. 7074; Cell Signaling Technology, Inc., Danvers, MA, USA) at room temperature for $1 \mathrm{~h}$. The specific proteins were measured using an enhanced chemiluminescence Western blotting kit (cat. no. RPN2108; GE Healthcare, Chicago, IL, USA) according to the instructions from the manufacturer. Antibodies against FLVCR1 (cat. no. PA5-28463; 1:1,000) was purchased from Invitrogen (Thermo Fisher Scientific, Inc.). Microtubule associated protein 1 light chain $3 \beta$ (LC3; cat. no. L7543; 1:1,000) and nucleoporin p62 (cat. no. N1163; 1:1,000) antibodies were purchased from Sigma-Aldrich (Merck KGaA, Darmstadt, Germany). An internal control GAPDH (cat. no. sc-47724; 1:1,000) or $\beta$-actin (cat. no. sc-8432; 1:1,000) antibodies were purchased from Santa Cruz Biotechnology, Inc. (Dallas, TX, USA). The quantitative densitometry analysis of immunoreactive band was performed using ImageJ bundled with 64-bit Java 1.6.0_24 program for Windows from the National Institutes of Health (Bethesda, MD, USA).

MTT assay. Cell viability was determined via MTT assay as previously reported (24). Briefly, cells were plated in 96-well plates at initial density of $2 \times 10^{3}$ cells/well. At each time point, cells were stained with $100 \mathrm{ml}$ sterile MTT dye $\left(0.5 \mathrm{mg} / \mathrm{ml}\right.$; Sigma-Aldrich; Merck KGaA) for $4 \mathrm{~h}$ at $37^{\circ} \mathrm{C}$, followed by removal of the culture medium and addition of $100 \mathrm{ml}$ of dimethyl sulfoxide (Sigma-Aldrich; Merck KGaA). The optical density (OD) was measured at $490 \mathrm{~nm}$ using an EL-311SX ELISA reader (BioTek Instruments, Inc., Winooski, Vermont, USA). All experiments were performed in triplicate.

Colony formationassay. Colony formation assay was performed as described previously (7). Briefly, cells were seeded in 6-well plates $\left(5 \times 10^{2}\right.$ cells) and maintained for 10 days. The colonies were stained with $1 \%$ crystal violet for $30 \mathrm{sec}$ after fixation with $4 \%$ formaldehyde (both from Sigma-Aldrich; Merck $\mathrm{KGaA}$ ) for $5 \mathrm{~min}$. Colonies were counted and the results were presented as the fold change compared to vector control cells.
Flow cytometry analysis of the cell cycle and apoptosis. Cell cycle and apoptosis analysis by using flow cytometry were performed as previously reported $(7,25)$. Cells were seeded in 6 -well plates. Following trypsinization, cells were centrifuged at $400 \mathrm{x} \mathrm{g}$ for $5 \mathrm{~min}$ at $4^{\circ} \mathrm{C}$, rinsed twice with ice cold PBS, and fixed at $4^{\circ} \mathrm{C}$ in $70 \%$ alcohol for $>1 \mathrm{~h}$. Subsequently, fixed cells were resuspended in PBS containing 0.5\% Triton X-100 and $100 \mu \mathrm{g} / \mathrm{ml}$ RNase on ice for $30 \mathrm{~min}$, and stained with $50 \mu \mathrm{g} / \mathrm{ml}$ propidium iodide (PI; Sigma-Aldrich; Merck KGaA) in the dark for $30 \mathrm{~min}$. Cells were analyzed for cell cycle distribution using a FACScan flow cytometer and BD ModFit software (both from BD Biosciences, San Jose, CA, USA) was used to analyze the data in accordance with the manufacturer's guidelines.

For apoptosis analysis, the same procedures were conducted as those for cell cycle assay described above and cell apoptosis was detected using Annexin V-FITC/PI Apoptosis Detection kit (cat. no. BMS500FI-100; eBioscience; Thermo Fisher Scientific, Inc.) and analyzed with to a FACScan flow cytometer (BD Biosciences), according to the manufacturer's protocol.

Xenografted tumor model. The xenografted tumor model was performed as previously described (7). For the SS xenograft model, 4-week-old NOD/SCID nude mice (14-20 g; Vital River Laboratory Animal Technology Co., Ltd., Beijing, China) were housed in a temperature-controlled, pathogen-free environment and used for experimentation. All experimental procedures were approved by the Institutional Animal Care and Use Committee of Shandong University (Jinan, China). The mice were randomly divided into two groups $(n=4$ per group), and HS-SY-II cells $\left(2 \times 10^{6}\right)$ transduced with lentivirus expressing shFLVCR1 or negative control vector were subcutaneously injected into the right flank of nude mice. Tumor length $(\mathrm{L})$, width $(\mathrm{W})$ and diameter were measured every week from week 1 to week 6; tumor volume $\left(\mathrm{mm}^{3}\right)$ was calculated using the following formula: $\mathrm{L} \mathrm{x} \mathrm{W}^{2} / 2(7,26)$. After 6 weeks, mice were sacrificed and tumors were harvested.

Statistical analysis. Data are presented as the mean \pm standard deviation from at least three separate experiments. Statistical analysis was performed with one-way analysis of variance with post hoc Bonferroni testing for multiple comparison or Student's t-test using GraphPad Prism 6 software (GraphPad Software Inc., La Jolla, CA, USA). P $<0.05$ was considered to indicate a statistically significant difference.

\section{Results}

FLVCR1 is upregulated in human SS cells and tissues. Gene and protein expression analysis in SW982 and HS-SY-II SS cell lines demonstrated that FLVCR1 was markedly upregulated in SS cell lines compared with synovialcytes derived from patients with osteoarthritis (Fig. 1A and B). Additionally, in eight pairs of human SS tissues, the mRNA and protein levels of FLVCR1 were higher than those in matched adjacent nontumor tissues (Fig. 1C and D). In accordance with the western blot results, the protein levels of FLVCR1 in eight specimens measured by immunohistochemistry further demonstrated higher expression of FLVCR1 in SS tissues than 
A

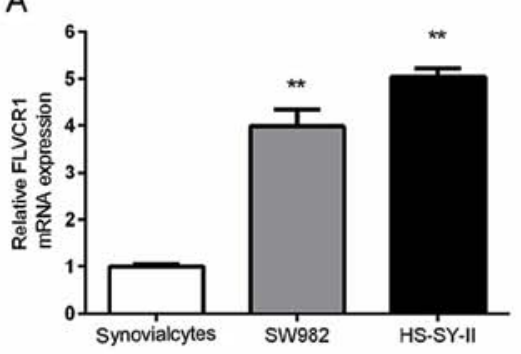

B

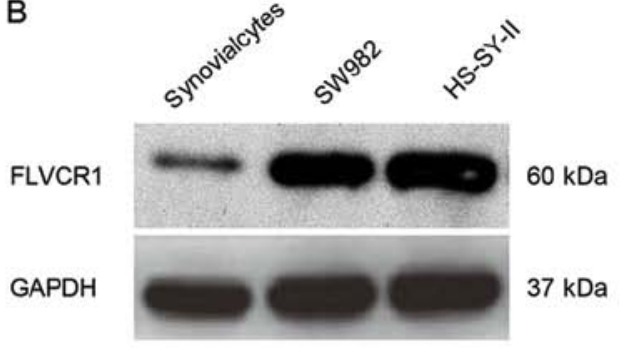

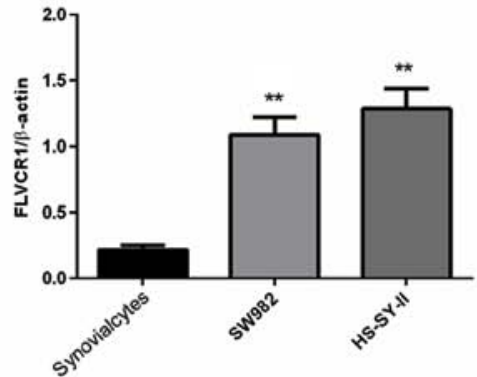

C

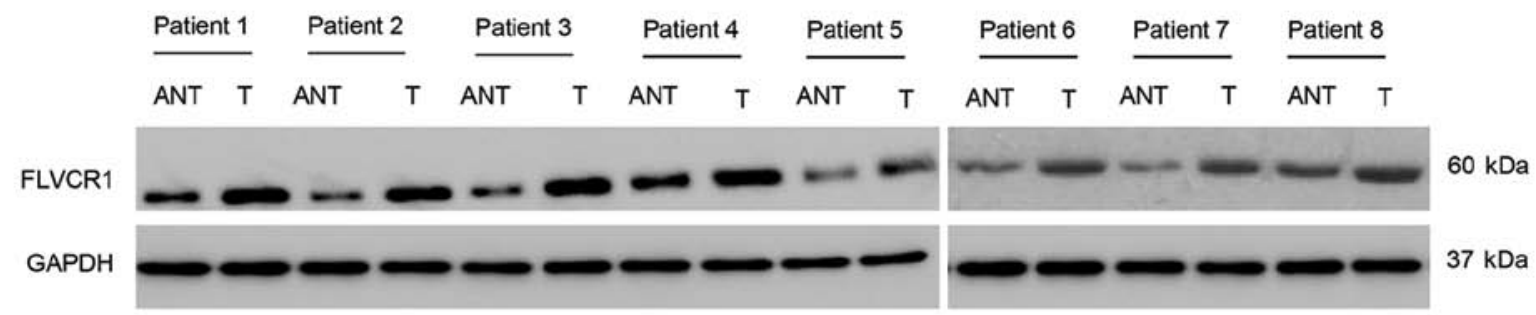

D

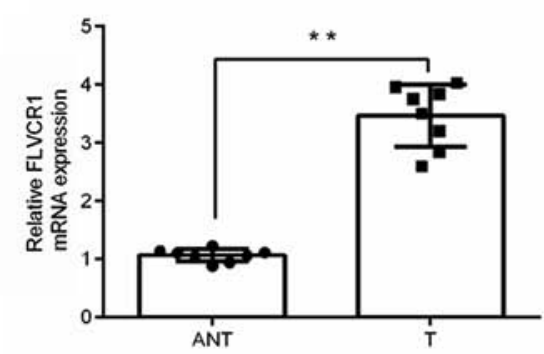

E

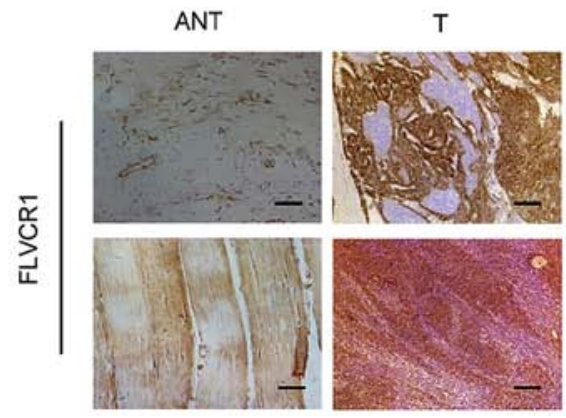

Figure 1. Expression of FLVCR1 is increased in SS cells and SS tissues. (A) RT-qPCR and (B) western blot analysis of FLVCR1 expression in SS cells. ${ }^{* *} \mathrm{P}<0.01$ vs. synovialcytes. Expression of FLVCR1 in SS tissues and ANT samples determined by (C) western blot, (D) qPCR and (E) immunohistochemistry (magnification, x100; scale bar, $50 \mu \mathrm{m}$ ). SS, synovial sarcoma; RT-qPCR, reverse transcription-quantitative polymerase chain reaction; FLVCR1, feline leukemia virus subgroup C cellular receptor 1; ANT, adjacent nontumor tissue; T, tumor.

those in matched adjacent nontumor tissues (Fig. 1E). These data collectively suggested that FLVCR1 expression is upregulated in SS.

Silencing of FLVCR1 expression in SS cells by lentivirus mediated RNA interference. To determine the potential roles of FLVCR1 in SS, FLVCR1 was silenced in the SS cell lines, SW982 and HS-SY-II, through lentivirus-mediated gene infection. As demonstrated in Fig. 2A, the majority of SW982 and HS-SY-II cells were GFP-positive following infection with lentivirus expressing shRNA targeting FLVCR1 (Lv-shFLVCR1) or Lv-shCon, indicating that the SS cells were effectively infected by the recombinant lentivirus. As demonstrated in Fig. 2B and C, the gene and protein levels of FLVCR1 in Lv-shFLVCR1 infected SS cells were significantly suppressed, further corroborating the knockdown efficiency. These findings demonstrated that recombinant lentivirus carrying shFLVCR1 effectively inhibited the expression of endogenous FLVCR1 in SS cells.

Silencing of FLVCR1 reduces the proliferation and colony formation ability of SS cells. To investigate the specific role of FLVCR1 in the proliferation and colony formation ability of SS cells, a 5-day MTT and colony formation assay were performed, respectively. Fig. 3A demonstrates that Lv-shFLVCR1 infected SS cells exhibited a slower growth rate compared with Lv-shCon infected cells. On day 5, the OD490 of Lv-shFLVCR1 infected SW982 and HS-SY-II cells were $0.63 \pm 0.02$ and $0.52 \pm 0.01$, respectively, whereas they were $0.79 \pm 0.03$ and $0.64 \pm 0.01$ in the $\mathrm{Lv}$-shCon infected cells. Consistently, colony formation assay further confirmed these results, Lv-shFLVCR1 infected SW982 and HS-SY-II cells produced less $(32.0 \pm 3.0$ and $15.67 \pm 3.21)$ and smaller colonies compared with $\mathrm{Lv}$-shCon infected cells $(81.67 \pm 5.51$ and $60.67 \pm 9.86$ ) (Fig. 3B). Thus, these data suggested that FLVCR1 promoted proliferation and colony formation of SS cells.

Inhibition of FLVCR1 impairs cell cycle progression of SS cells. To further investigate the underlying mechanisms of inhibition of cell proliferation and colony formation, cell cycle phases of SS cells were detected using PI staining and flow cytometry following FLVCR1 knockdown. As presented in Fig. 4A, $53.96 \pm 1.14$ and $40.54 \pm 1.78 \%$ of cells were at the G0/G1 phase in Lv-shFLVCR1-infected SW982 and HS-SY-II cells, which was lower than Lv-shCon-infected SW982 $(66.70 \pm 1.68 \%)$ and HS-SY-II cells $(49.85 \pm 0.51 \%)$. Additionally, there were significantly more cells at $\mathrm{S}$ phase following Lv-shFLVCR1 infection in SW982 and HS-SY-II cells (32.69 \pm 4.77 and $30.81 \pm 1.22 \%$ ), 
A

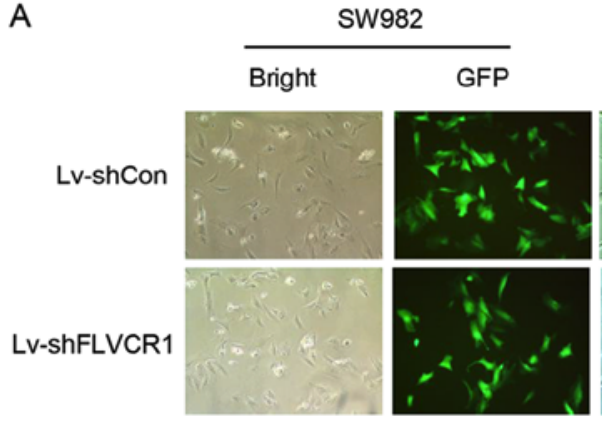

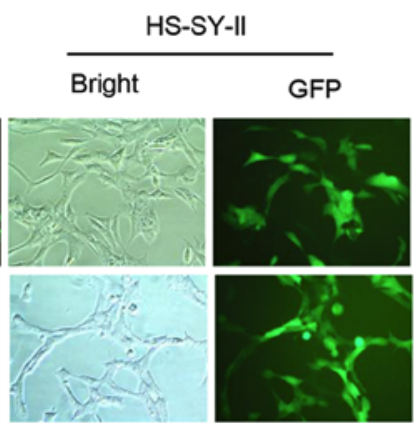

B

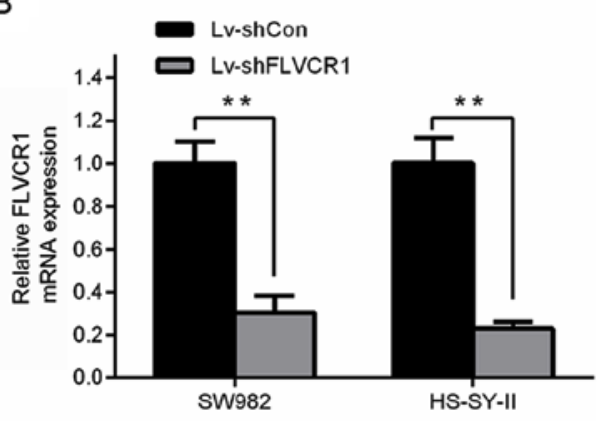

C

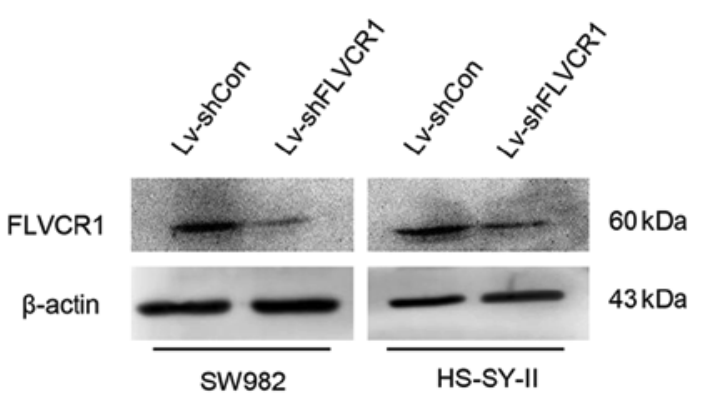

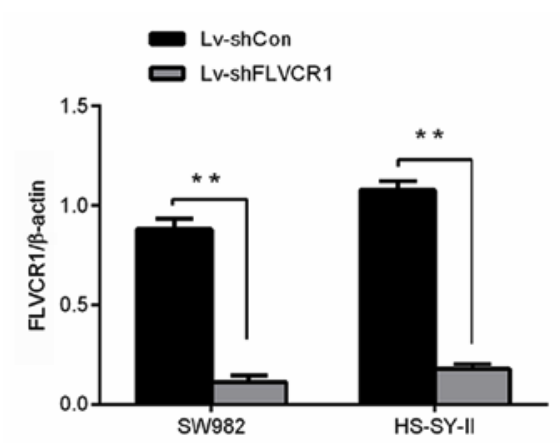

Figure 2. FLVCR1 silencing efficiency by shRNA lentivirus. (A) Representative images of Lv-shCon and Lv-shFLVCR1 infected SS cells under fluorescence microscope. (B) FLVCR1 expression in Lv-shCon and Lv-shFLVCR1 infected SS cells analyzed by RT-qPCR. (C) Protein levels of FLVCR1 in Lv-shCon and Lv-shFLVCR1 infected SS cells determined by western blotting. ${ }^{* *} \mathrm{P}<0.01$. SS, synovial sarcoma; GFP, green fluorescent protein; Lv-sh, lentiviral short hairpin RNA; Con, control; FLVCR1, feline leukemia virus subgroup C cellular receptor 1.
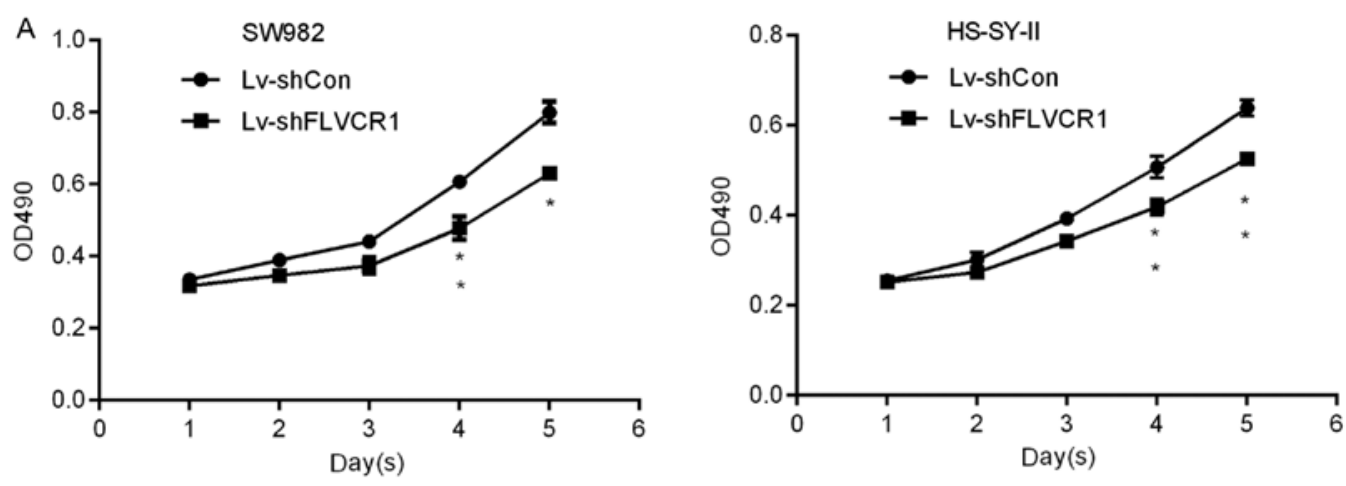

B
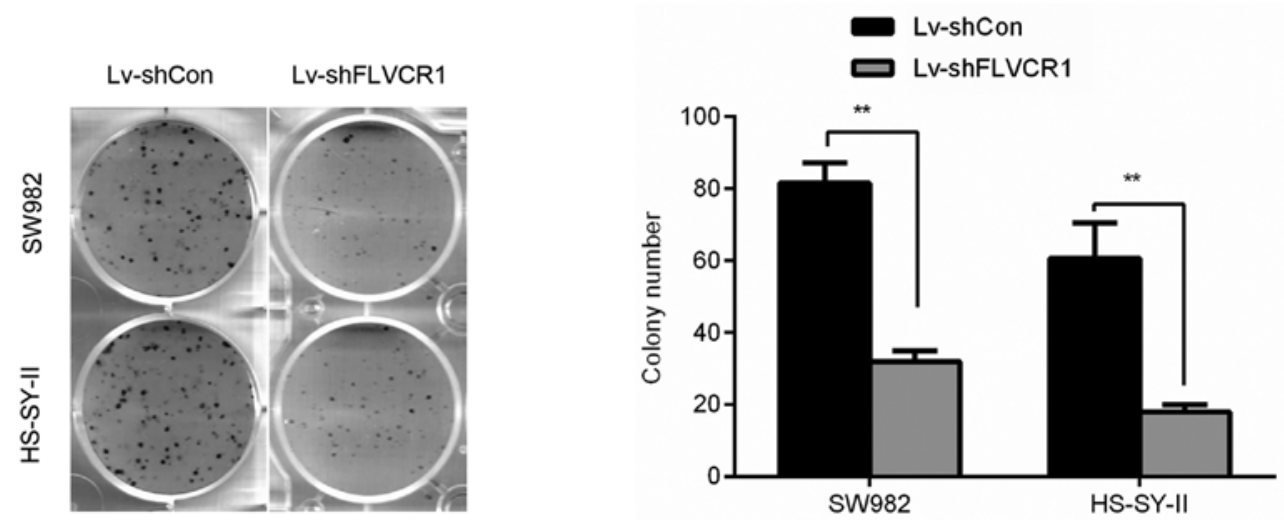

Figure 3. Silencing of FLVCR1 inhibits cell proliferation and colony formation ability of synovial sarcoma cells. (A) Cell proliferation determined by MTT assay in SW982 and HS-SY-II cells. (B) Colony formation assays of Lv-shCon and Lv-shFLVCR1 infected SW982 and HS-SY-II cells. "P<0.05, ${ }^{* *} \mathrm{P}<0.01$ vs. Lv-shCon. OD, optical density; Lv-sh, lentiviral short hairpin RNA; Con, control; FLVCR1, feline leukemia virus subgroup C cellular receptor 1.

compared with Lv-shCon infected SW982 and HS-SY-II cells $(20.78 \pm 1.45$ and $26.14 \pm 1.02 \%)$ (Fig. 4A). These results suggested that cell cycle progression was impaired in FLVCR1-silenced SS cells. Furthermore, $18.36 \pm 2.34$ and $2.84 \pm 0.04 \%$ cells were in the 

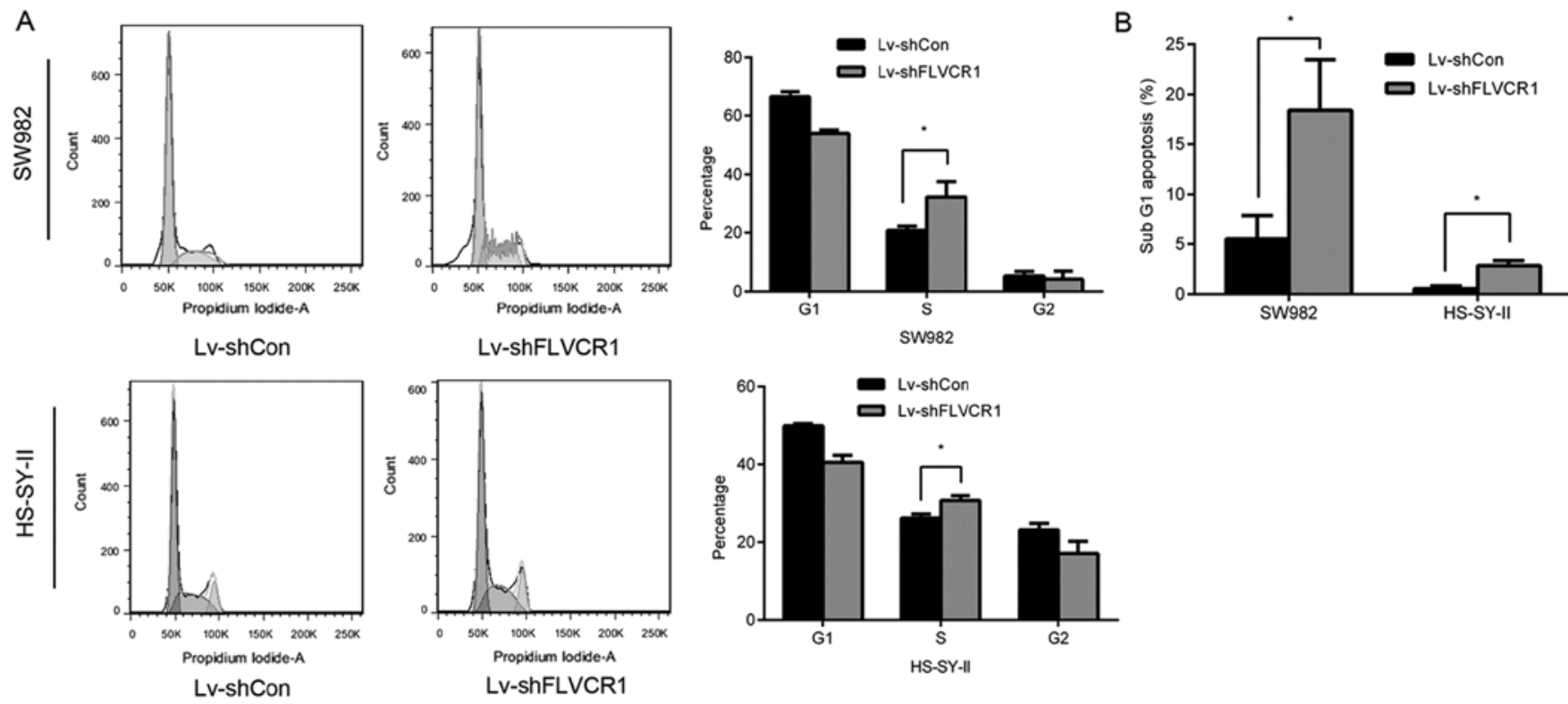

Figure 4. Silencing of FLVCR1 impairs cell cycle progression of synovial sarcoma cells. (A) Representative plots of flow cytometry analysis of SW982 and HS-SY-II cell cycle using propidium iodide staining. The bar graph presents statistic analysis of percentages of SW982 and HS-SY-II cells at different cell cycle stages (G0/G1, S and G2/M). (B) Statistic analysis of percentages of sub-G1 SW982 and HS-SY-II cells. "P<0.05. Lv-sh, lentiviral short hairpin RNA; Con, control; FLVCR1, feline leukemia virus subgroup C cellular receptor 1.

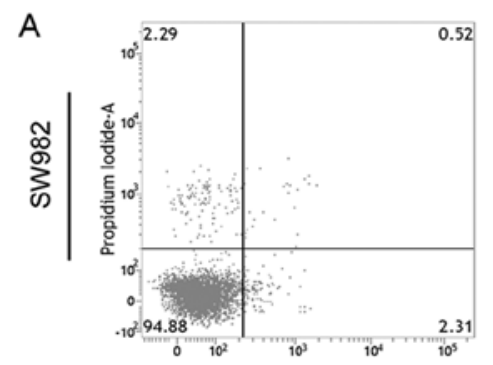

Lv-shCon

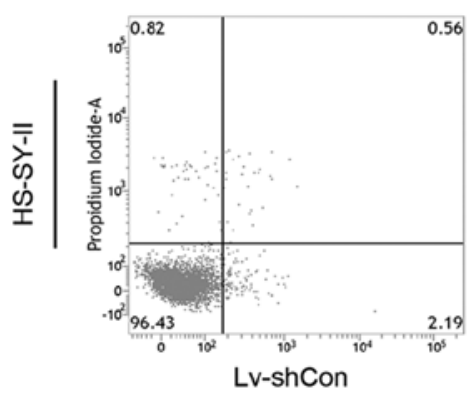

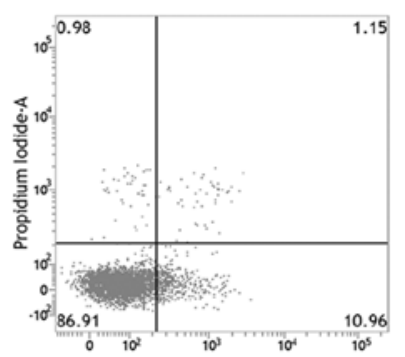

Lv-ShFLVCR1

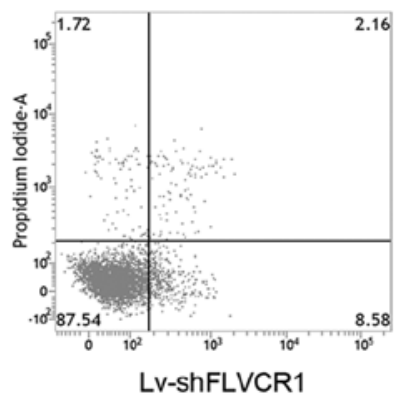

B

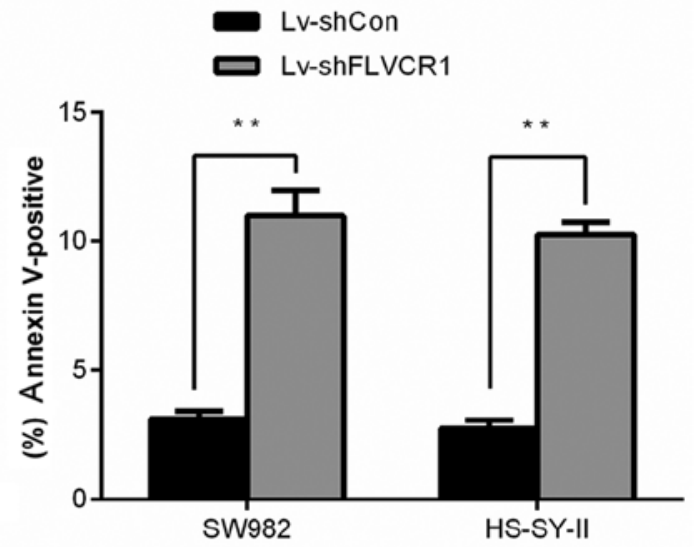

Figure 5. Silencing of FLVCR1 increases apoptosis of synovial sarcoma cells. (A) Flow cytometry analysis showed knockdown the expression of FLVCR1 in SW982 and HS-SY-II cells increased apoptosis. (B) Bar graphs present percentage of the early (lower right) and late apoptotic (upper right) cells. ** $\mathrm{P}<0.01$. Lv-sh, lentiviral short hairpin RNA; Con, control; FLVCR1, feline leukemia virus subgroup C cellular receptor 1.

sub-G1 phase in Lv-shFLVCR1-infected SW982 and HS-SY-II cells, higher in Lv-shCon-infected SW982 and HS-SY-II cells $(5.52 \pm 0.05$ and $0.55 \pm 0.02 \%$ ) (Fig. 4B), suggesting that there were more apoptotic cells in the group with FLVCR1 suppression.

Silencing of FLVCR1 induced apoptosis of SS cells. As presented in Fig. 5, the percentage of early and late apoptotic SW982 and HS-SY-II cells increased to $10.99 \pm 0.97$ and $10.26 \pm 0.48 \%$, respectively, in Lv-shFLVCR1 cells, which was significantly higher than that of the control $(3.12 \pm 0.29$ and $2.74 \pm 0.33 \%$ ), indicating FLVCR1 silencing promoted SS cell apoptosis.

Silencing of FLVCR1 induced cytotoxic autophagy of SS cells. To assess whether FLVCR1 affects autophagy, the expression of LC3-II, LC3-I and nucleoporin p62 (a known autophagy substrate) in SS cells was assayed by western blot, and the LC3-II/LC3-I ratio (a specific indicator of autophagy level) was calculated. As presented in Fig. 6, an increased accumulation 
A
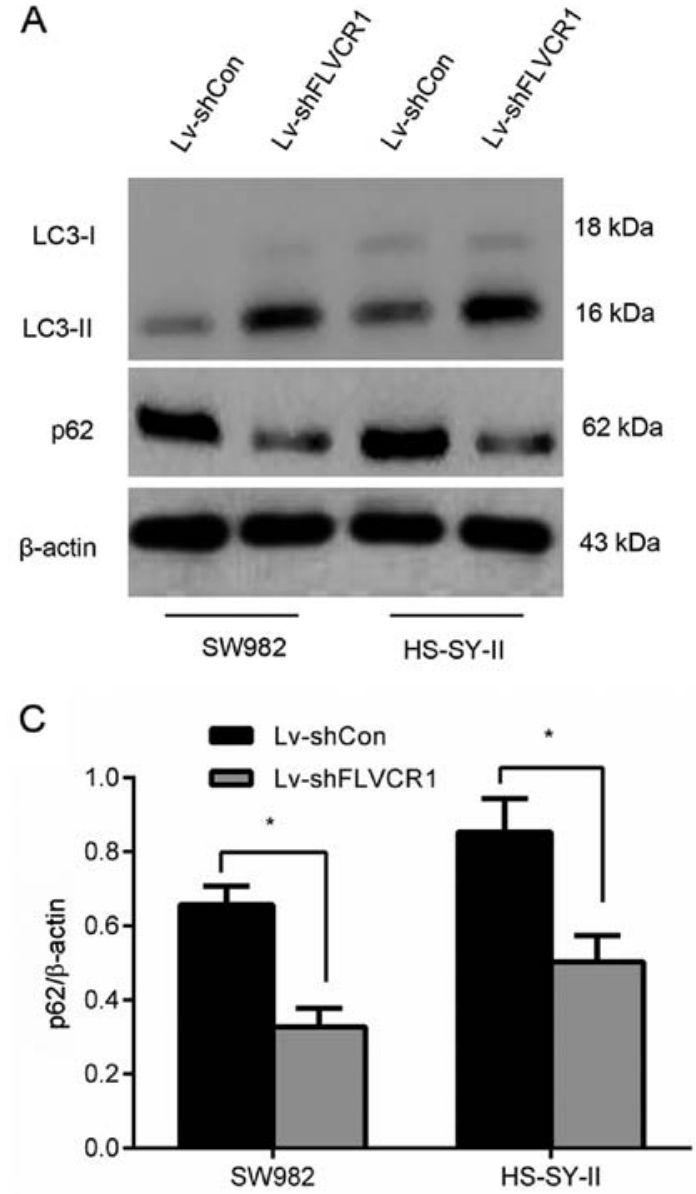

B

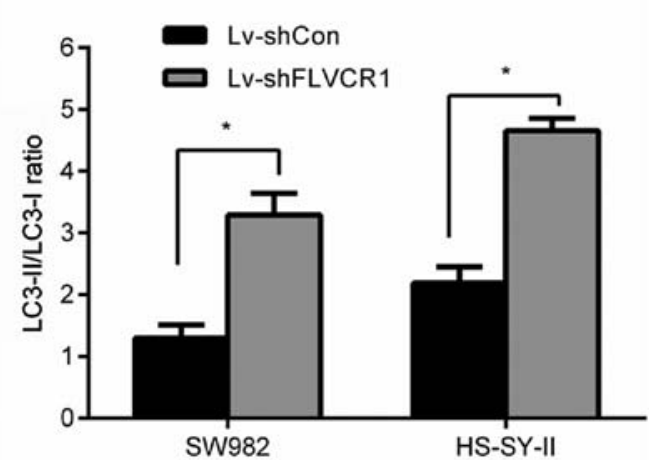

Figure 6. Silencing of FLVCR1 induces cytotoxic autophagy of synovial sarcoma cells. (A) Accumulation of LC3-II and degradation of nucleoporin p62 (a known autophagy substrate) determined by western blot. (B) Ratio of LC3-II/LC3-I and (C) p62/3-actin when treated with Lv-shFLVCR1 for 72 h determined by densitometry analysis of western blots. "P<0.05. Lv-sh, lentiviral short hairpin RNA; Con, control; FLVCR1, feline leukemia virus subgroup C cellular receptor $1 ; \mathrm{LC} 3$, microtubule associated protein 1 light chain $3 \beta$.

of LC3-II and reduced expression of p62 were observed in Lv-shFLVCR1-infected cells compared to those in Lv-shConinfected cells. Collectively, the results suggest that silencing of FLVCR1 induced cytotoxic autophagy, thus, promoting apoptosis under certain conditions of metabolic stress.

Silencing of FLVCRI suppressed SS cell growth in vivo. A subcutaneous SS nude mouse xenograft model was established to confirm whether silencing of FLVCR1 inhibited the growth of SS cells in vivo. As displayed in Fig. 7A and B, the mouse group infected with Lv-shFLVCR1 lentivirus had a lower proliferation rate, and formed markedly smaller tumors than the Lv-shCon lentivirus group. The tumor size at the time of sacrifice in the Lv-shFLVCR1 lentivirus group was $375 \pm 40.7 \mathrm{~mm}^{3}$, which was significantly smaller than in the $\mathrm{Lv}$-shCon lentivirus group $\left(1,067 \pm 95.2 \mathrm{~mm}^{3}\right)$. Additionally, in agreement with the above in vitro results, reduced expression of FLVCR1 mRNA (Fig. 7C) and protein (Fig. 7D) were observed in tumor tissues following the inhibition of FLVCR1 compared with control tumor tissues.

It was also investigated whether Lv-shFLVCR1 induces cytotoxic autophagy of SS cells in vivo. Western blot analysis revealed increased accumulation of LC3-II and degradation of p62 in Lv-shFLVCR1 tumors, as compared with Lv-shCon tumors (Fig. 7E). These results, taken together, suggest that
FLVCR1 silencing suppressed SS tumorigenicity in vivo through, partially inducing cytotoxic autophagy.

\section{Discussion}

The current study investigated a novel role for FLVCR1 in promoting the proliferation and tumorigenicity of SS, which has not been reported previously, to the best of our knowledge. The data revealed that FLVCR1 enhances the proliferation and tumorigenicity of SS cells in vitro and in vivo. FLVCR1 silencing reduced the proliferation and tumorigenicity of SS cells via effects on the cell cycle and autophagy. The results from the current study provided novel insights and evidence that FLVCR1 play a crucial role in promoting the tumorigenicity and progression of human SS, indicating that targeting FLVCR1 may be useful as a novel therapeutic strategy in the treatment of patients with SS.

Overexpression of FLVCR1 in human SS cells suggested that FLVCR1 may have a key role in the development and progression of SS. To examine its roles, lentivirus-mediated knockdown was used to specifically suppress FLVCR1 gene expression. MTT and colony formation assays demonstrated that silencing of FLVCR1 significantly inhibited cell proliferation and colony formation compared with control cells. Through flow cytometry analysis, it was demonstrated that 
A

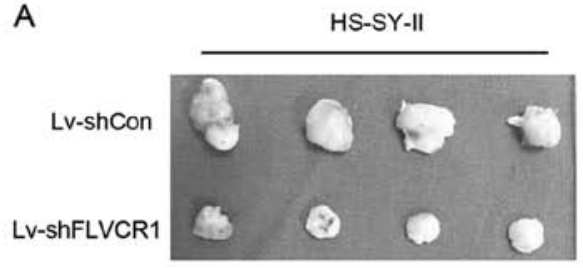

D

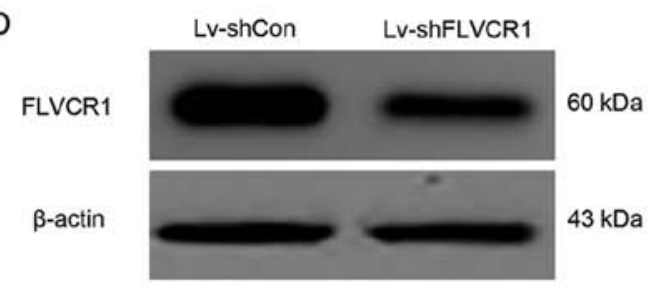

$E$

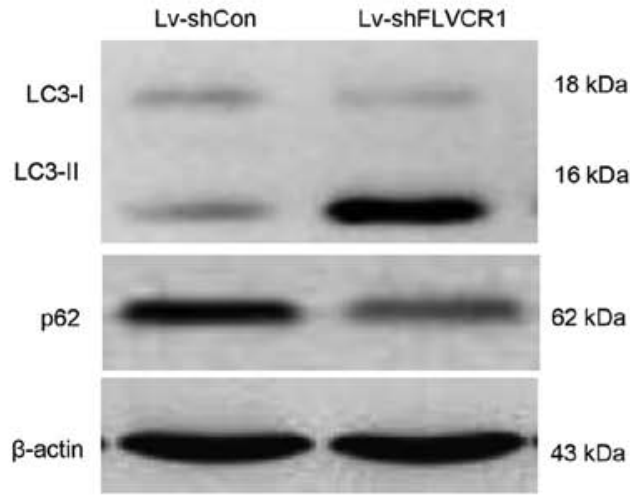

B
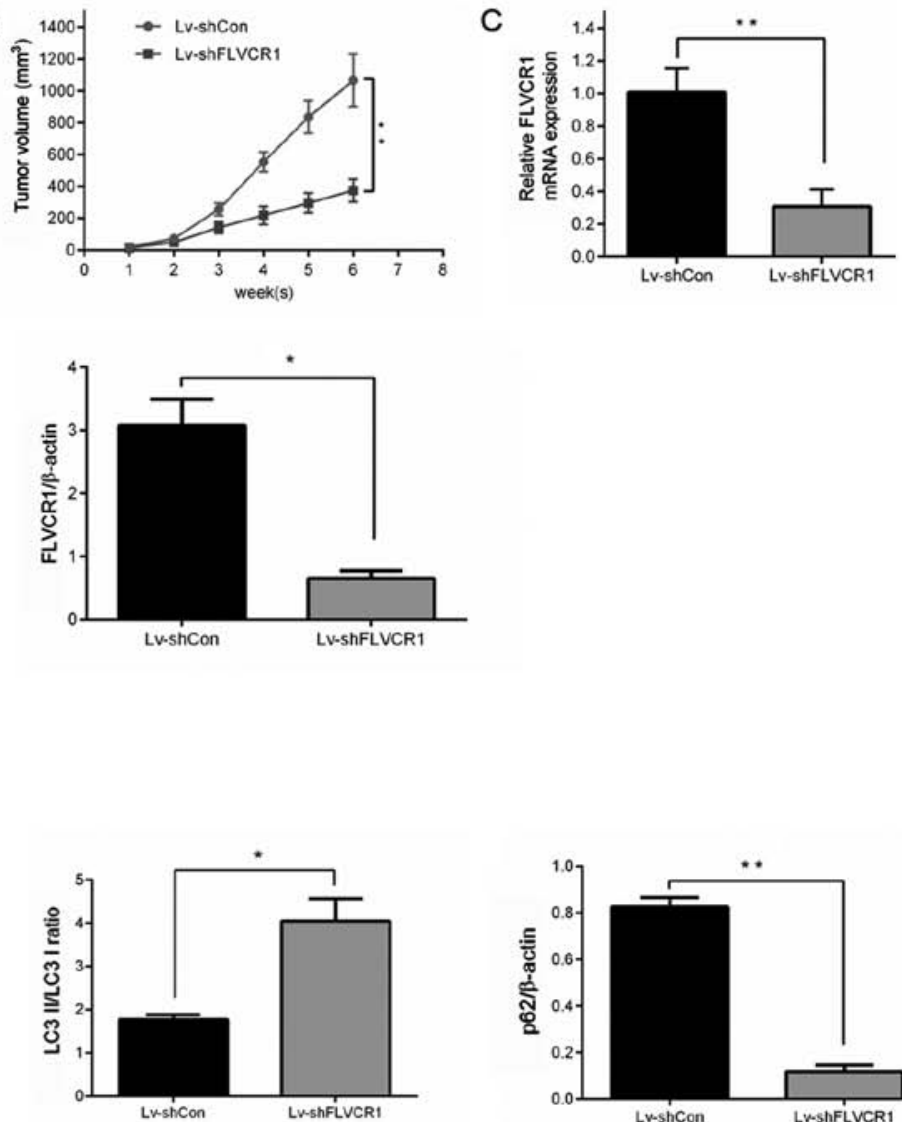

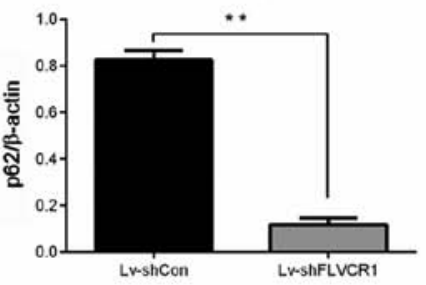

Figure 7. Inhibition of FLVCR1 suppresses synovial sarcoma tumor growth in vivo. (A) Representative images of xenograft tumors. (B) Tumor volume growth curve. (C) FLVCR1 mRNA and (D) protein level in the Lv-shFLVCR1 treatment group in vivo compared with the Lv-shCon group as determined by reverse transcription-quantitative polymerase chain reaction and western blot, respectively. (E) Effect of downregulation of FLVCR1 on LC3-II and degradation of nucleoporin p62 in vivo. ${ }^{*} \mathrm{P}<0.05$ and ${ }^{* *} \mathrm{P}<0.01$. Lv-sh, lentiviral short hairpin RNA; Con, control; FLVCR1, feline leukemia virus subgroup C cellular receptor $1 ; \mathrm{LC} 3$, microtubule associated protein 1 light chain $3 \beta$.

downregulation of FLVCR1 resulted in impaired cell cycle progression and increased apoptosis in SW982 and HS-SY-II cells. The results suggested that the observed growthpromoting effect of FLVCR1 on SS cells may be mediated through modulation of cell cycle progression. This pro-growth role of FLVCR1 is similar to a previous study, which revealed that FLVCR1 is important for the survival of neuroblastoma cells (15). Notably, in vivo studies demonstrated that FLVCR1 knockdown significantly suppressed the growth of SS in a xenograft mouse model. Clearly, findings from the present study have highlighted the important role of FLVCR1 in tumor growth, indicating its potential as a novel therapeutic target.

Previous studies demonstrated that depletion of FLVCR1 did not affect the cell cycle of K562 leukemia cells and Caco2 colon carcinoma cells $(18,19)$; however, in the current study, FLVCR1 silencing impaired cell cycle progression of SS cell lines. A number of factor may account for the differences in the effects on cell cycle arrest in the current study compared with previous studies: i) The requirement for FLVCR1 in cell cycle progression may vary in different cancer cell lines due to differences in mechanisms to bypass cell cycle arrest; ii) the G1 arrest observed in K562 and Caco2 cells with FLVCR1 knockdown, occurred when cells were concurrently treated with sodium butyrate $(18,19)$, raising the possibility that the arrest may be an indirect effect; and iii) variations in the degree of FLVCR1 knockdown may result in elaboration of different cell cycle phenotypes. For example, such differences have been observed with DNA methyltransferase 1 (DNMT1) depletion, where different levels of DNMT1 loss result in varying cell cycle effects (27).

Apoptosis and autophagy are two key mechanisms controlling cell survival and cell death $(21,28)$. The important role of apoptosis (the first known programmed cell death mechanism) in the pathogenesis of various diseases has been well demonstrated. As an important homeostatic mechanism, autophagy removes unnecessary or damaged proteins and dysfunctional cellular organelles, and recycles cytoplasmic contents in all living cells (29). Autophagy initially was described as a cell survival mechanism in response to stress (30), and in certain circumstances, autophagy may contribute to cell survival by averting apoptosis (31). Autophagy is closely associated with the apoptosis induced by hypoxia $(32,33)$. It has previously been reported that hypoxia modulates FLVCR1 gene expression via key transcription regulatory proteins, including HIF2 $\alpha$ and ETS1 (13). Additionally, FLVCR1 has a vital role in the survival of committed erythroid progenitors and 
neuroblastoma cells $(10,15)$. Based on these findings and the results of the current study in SS cells, we hypothesized that FLVCR1 may affect survival of SS cells via regulation of autophagy. Indeed, to the best of out knowledge, the present study was the first to demonstrate that knocking down FLVCR1 expression induces autophagy of SS cells in vitro and in vivo. Thus, the results demonstrated that the inhibition of growth in SS cells by FLVCR1 depletion may be, at least in part, due to the induction of autophagy.

Notably, the finding of the current study regarding autophagy are in contrast to what was reported by Petrillo et al (34), where western blot analysis exhibited no elevated LC3-II levels in CD31 ${ }^{+}$FLVCR1-knockout endothelial cells compared with FLVCR1-null endothelial cells. SS is a heterogeneous tumor, which exhibits different morphological and phenotypic profiles, including variation in cellular morphology, gene expression, metabolism, motility, proliferation and metastatic potential. Thus, the observed difference between the data of the current study and the previous study is potentially attributable to differences in the cell lines used. In addition, the effect of autophagy varies in different diseases, even in different time periods of one specific disease model (35).

Cancer development and progression is a complex process that involves a host of functional and genetic abnormalities, with various signaling pathways, including Wnt, Akt, SRC, MAPK, IGF-1R and TGF- $\beta$ signaling, implicated in the development of SS $(2,7,23,36)$. Whether FLVCR1 expression can affect these signaling pathways is largely unknown. Thus, the association between FLVCR1 and these signaling pathways in SS cell proliferation and metastasis required further investigation.

Use of the Basic Local Alignment Search Tool (blast. ncbi.nlm.nih.gov/Blast.cgi) demonstrated that the portion of FLVCR1 mRNA targeted by shRNA in the current study is the common area of FLVCR1b and FLVCRla. Thus, it is unclear which isoform of FLVCR1 was involved in promoting the proliferation and tumorigenicity of SS. Additionally, only the silencing effects of FLVCR1 were investigated in the present study; the effects of FLVCR1 overexpression on the tumorigenicity of SS will be addressed in future experiments.

In conclusion, the findings of the current study demonstrated that silencing of FLVCR1 expression by shRNA knockdown significantly inhibited the proliferation and tumorigenicity of $\mathrm{SS}$ cells in vitro and in vivo. In addition, FLVCR1 may affect survival of SS cells via regulation of autophagy, although the underlying molecular mechanism requires further investigation. The novel role of FLVCR1 in promoting tumorigenicity of SS cells suggests that it may be a potential target gene for treatment of patients with SS.

\section{Acknowledgements}

Not applicable.

\section{Funding}

This study was funded by the National Natural Science Foundation of China (grant no. 81301727) and the Youth Foundation of the Second Hospital of Shandong University (grant no. 2018YT28).

\section{Availability of data and materials}

The datasets used and/or analyzed during the current study are available from the corresponding author on reasonable request.

\section{Authors' contributions}

CLP and YS conceived and designed the study. XLL, FW, DJW, SZM, and XWW performed the experiments. WC, XYW, and CZG analyzed and interpreted the data. CLP and YS wrote the manuscript. All authors read and approved the final manuscript.

\section{Ethics approval and consent to participate}

Samples were obtained with patients' informed consent. All research involving human participants was been approved by the Second Hospital of Shandong University ethics committees (Jinan, China) and in accordance with the Declaration of Helsinki. All experimental procedures using animals were approved by the Institutional Animal Care and Use Committee of Shandong University (Jinan, China).

\section{Consent for publication}

Not applicable.

\section{Competing interests}

The authors declare that they have no competing interests.

\section{References}

1. Haldar M, Hancock JD, Coffin CM,Lessnick SL and Capecchi MR: A conditional mouse model of synovial sarcoma: Insights into a myogenic origin. Cancer Cell 11: 375-388, 2007.

2. Trautmann M, Sievers E, Aretz S, Kindler D, Michels S, Friedrichs N, Renner M, Kirfel J, Steiner S, Huss S, et al: SS18-SSX fusion protein-induced $\mathrm{Wnt} / \beta$-catenin signaling is a therapeutic target in synovial sarcoma. Oncogene 33: 5006-5016, 2014.

3. Herzog CE: Overview of sarcomas in the adolescent and young adult population. J Pediatr Hematol Oncol 27: 215-218, 2005.

4. Lewis JJ, Antonescu CR, Leung DH, Blumberg D, Healey JH, Woodruff JM and Brennan MF: Synovial sarcoma: A multivariate analysis of prognostic factors in 112 patients with primary localized tumors of the extremity. J Clin Oncol 18: 2087-2094, 2000.

5. Ladanyi M, Antonescu CR, Leung DH, Woodruff JM, Kawai A, Healey JH, Brennan MF, Bridge JA, Neff JR, Barr FG, et al: Impact of SYT-SSX fusion type on the clinical behavior of synovial sarcoma: A multi-institutional retrospective study of 243 patients. Cancer Res 62: 135-140, 2002.

6. Palmerini E, Staals EL, Alberghini M, Zanella L, Ferrari C, Benassi MS, Picci P, Mercuri M, Bacci G and Ferrari S: Synovial sarcoma: Retrospective analysis of 250 patients treated at a single institution. Cancer 115: 2988-2998, 2009.

7. Peng C, Zhao H, Chen W, Song Y, Wang X, Li J, Qiao Y, Wu D, Ma S, Wang X, et al: Identification of SHCBP1 as a novel downstream target gene of SS18-SSX1 and its functional analysis in progression of synovial sarcoma. Oncotarget 7: 66822-66834, 2016.

8. Qi Y, Wang N, He Y, Zhang J, Zou H, Zhang W, Gu W, Huang Y, Lian $\mathrm{X}, \mathrm{Hu} \mathrm{J}$, et al: Transforming growth factor- $\beta 1$ signaling promotes epithelial-mesenchymal transition-like phenomena, cell motility, and cell invasion in synovial sarcoma cells. PLoS One 12: e0182680, 2017.

9. Przybyl J, Jurkowska M, Rutkowski P, Debiec-Rychter M and Siedlecki JA: Downstream and intermediate interactions of synovial sarcoma-associated fusion oncoproteins and their implication for targeted therapy. Sarcoma 2012: 249219, 2012. 
10. Chiabrando D, Marro S, Mercurio S, Giorgi C, Petrillo S, Vinchi F, Fiorito V, Fagoonee S, Camporeale A, Turco E, et al: The mitochondrial heme exporter FLVCR1b mediates erythroid differentiation. J Clin Invest 122: 4569-4579, 2012.

11. Keel SB, Doty RT, Yang Z, Quigley JG, Chen J, Knoblaugh S, Kingsley PD, De Domenico I, Vaughn MB, Kaplan J, et al: A heme export protein is required for red blood cell differentiation and iron homeostasis. Science 319: 825-828, 2008.

12. Quigley JG, Yang Z, Worthington MT, Phillips JD, Sabo KM, Sabath DE, Berg CL, Sassa S, Wood BL and Abkowitz JL: Identification of a human heme exporter that is essential for erythropoiesis. Cell 118: 757-766, 2004.

13. Fiorito V, Neri F, Pala V, Silengo L, Oliviero S, Altruda F and Tolosano E: Hypoxia controls Flvcrl gene expression in Caco2 cells through HIF2 $\alpha$ and ETS1. Biochim Biophys Acta 1839: 259-264, 2014.

14. Mercurio S, Petrillo S, Chiabrando D, Bassi ZI, Gays D, Camporeale A, Vacaru A, Miniscalco B, Valperga G, Silengo L, et al: The heme exporter Flvcrl regulates expansion and differentiation of committed erythroid progenitors by controlling intracellular heme accumulation. Haematologica 100: 720-729, 2015.

15. Chiabrando D, Castori M, di Rocco M, Ungelenk M, Giesselmann S, Di Capua M, Madeo A, Grammatico P, Bartsch S, Hübner CA, et al: Mutations in the heme exporter FLVCR1 cause sensory neurodegeneration with loss of pain perception. PLoS Genet 12: e1006461, 2016.

16. Rajadhyaksha AM, Elemento O, Puffenberger EG, Schierberl KC, Xiang JZ, Putorti ML, Berciano J, Poulin C, Brais B, Michaelides M, et al: Mutations in FLVCR1 cause posterior column ataxia and retinitis pigmentosa. Am J Hum Genet 87: 643-654, 2010.

17. Vinchi F, Ingoglia G, Chiabrando D, Mercurio S, Turco E, Silengo L, Altruda F and Tolosano E: Heme exporter FLVCR1a regulates heme synthesis and degradation and controls activity of cytochromes P450. Gastroenterology 146: 1325-1338, 2014.

18. Fiorito V, Forni M, Silengo L, Altruda F and Tolosano E: Crucial role of FLVCR1a in the maintenance of intestinal heme homeostasis. Antioxid Redox Signal 23: 1410-1423, 2015.

19. Mercurio S, Aspesi A, Silengo L, Altruda F, Dianzani I and Chiabrando D: Alteration of heme metabolism in a cellular model of Diamond-Blackfan anemia. Eur J Haematol 96: 367-374, 2016

20. Zeng Z, Lin H, Zhao X, Liu G, Wang X, Xu R, Chen K, Li J and Song L: Overexpression of GOLPH3 promotes proliferation and tumorigenicity in breast cancer via suppression of the FOXO1 transcription factor. Clin Cancer Res 18: 4059-4069, 2012.

21. Jiao G, Guo W, Ren T, Lu Q, Sun Y, Liang W, Ren C, Yang K and Sun K: BMPR2 inhibition induced apoptosis and autophagy via destabilization of XIAP in human chondrosarcoma cells. Cell Death Dis 5: e1571, 2014.

22. Livak KJ and Schmittgen TD: Analysis of relative gene expression data using real-time quantitative PCR and the 2(-Delta Delta C(T)) method. Methods 25: 402-408, 2001.

23. Peng $\mathrm{C}$, Zhao $\mathrm{H}$, Song $\mathrm{Y}$, Chen $\mathrm{W}$, Wang $\mathrm{X}$, Liu $\mathrm{X}$, Zhang $\mathrm{C}$, Zhao J, Li J, Cheng G, et al: SHCBP1 promotes synovial sarcoma cell metastasis via targeting TGF- $\beta 1 /$ Smad signaling pathway and is associated with poor prognosis. J Exp Clin Cancer Res 36 : $141,2017$.
24. Han RL, Wang FP, Zhang PA, Zhou XY and Li Y: miR-383 inhibits ovarian cancer cell proliferation, invasion and aerobic glycolysis by targeting LDHA. Neoplasma 64: 244-252, 2017.

25. Peng C, Guo W, Yang Y and Zhao H: Downregulation of SS18-SSX1 expression by small interfering RNA inhibits growth and induces apoptosis in human synovial sarcoma cell line HS-SY-II in vitro. Eur J Cancer Prev 17: 392-398, 2008.

26. Wang S, Liu H, Ren L, Pan Y and Zhang Y: Inhibiting colorectal carcinoma growth and metastasis by blocking the expression of VEGF using RNA interference. Neoplasia 10: 399-407, 2008.

27. Brown KD and Robertson KD: DNMT1 knockout delivers a strong blow to genome stability and cell viability. Nat Genet 39: 289-290, 2007.

28. Shimizu S, Kanaseki T, Mizushima N, Mizuta T, ArakawaKobayashi S, Thompson CB and Tsujimoto Y: Role of Bcl-2 family proteins in a non-apoptotic programmed cell death dependent on autophagy genes. Nat Cell Biol 6: 1221-1228, 2004.

29. Sun Y, Guo W, Ren T, Liang W, Zhou W, Lu Q, Jiao G and Yan T: Gli1 inhibition suppressed cell growth and cell cycle progression and induced apoptosis as well as autophagy depending on ERK1/2 activity in human chondrosarcoma cells. Cell Death Dis 5: e979, 2014.

30. Ogata M, Hino S, Saito A, Morikawa K, Kondo S, Kanemoto S, Murakami T, Taniguchi M, Tanii I, Yoshinaga K, et al: Autophagy is activated for cell survival after endoplasmic reticulum stress. Mol Cell Biol 26: 9220-9231, 2006.

31. Maiuri MC, Zalckvar E, Kimchi A and Kroemer G: Self-eating and self-killing: Crosstalk between autophagy and apoptosis. Nat Rev Mol Cell Biol 8: 741-752, 2007.

32. Vangamudi B, Paul TA, Shah PK, Kost-Alimova M, Nottebaum L, Shi X, Zhan Y,Leo E, Mahadeshwar HS, Protopopov A, et al: The SMARCA2/4 ATPase domain surpasses the bromodomain as a drug target in SWI/SNF-mutant cancers: Insights from cDNA rescue and PFI-3 inhibitor studies. Cancer Res 75: 3865-3878, 2015.

33. Fang Y, Tan $\mathbf{J}$ and Zhang Q: Signaling pathways and mechanisms of hypoxia-induced autophagy in the animal cells. Cell Biol Int 39: 891-898, 2015.

34. Petrillo S, Chiabrando D, Genova T, Fiorito V, Ingoglia G, Vinchi F, Mussano F, Carossa S, Silengo L, Altruda F, et al: Heme accumulation in endothelial cells impairs angiogenesis by triggering paraptosis. Cell Death Differ: Dec 11, 2017. (Epub ahead of print). doi: 10.1038/s41418-017-0001-7.

35. Wang L, Jin Z, Wang J, Chen S, Dai L, Lin D, Wu L and Gao W: Detrimental effect of hypoxia-inducible factor- $1 \alpha$-induced autophagy on multiterritory perforator flap survival in rats. Sci Rep 7: 11791, 2017.

36. Michels S, Trautmann M, Sievers E, Kindler D, Huss S, Renner M, Friedrichs N, Kirfel J, Steiner S, Endl E, et al: SRC signaling is crucial in the growth of synovial sarcoma cells. Cancer Res 73: 2518-2528, 2013 